\title{
Factores asociados a insuficiencia renal postoperatoria en cirugía de revascularización miocárdica
}

\author{
Albert F. Guerrero B. ${ }^{a, b, *}$, Jaime Camacho M. ${ }^{a}$, Néstor F. Sandoval ${ }^{c}$, \\ Juan P. Umaña M. ${ }^{\text {d }}$, Carlos E. Obando ${ }^{a}$ y Marisol Carreño ${ }^{a}$ \\ a Departamento de Cirugía Cardiovascular. Fundación Cardioinfantil - Instituto de Cardiología, Bogotá, Colombia \\ b Universidad Colegio Mayor Nuestra Señora del Rosario, Bogotá, Colombia \\ c Departamento de Cirugía Cardiovascular Pediátrica, Fundación Caridoinfantil-Instituto de Cardiología, Bogotá, Colombia \\ ' Dirección científica de Medicina cardiovascular y de alta complejidad, Fundación Cardioinfantil, Bogotá, Colombia
}

Recibido el 7 de mayo de 2015; aceptado el 4 de septiembre de 2015

Disponible en Internet el 4 de diciembre de 2015

\author{
PALABRAS CLAVE \\ Disfunción renal; \\ Cirugía de \\ revascularización \\ coronaria; \\ Enfermedad \\ coronaria
}

\begin{abstract}
Resumen
Objetivos: Determinar qué antecedentes clínicos del paciente y factores del procedimiento quirúrgico se asocian con la aparición de insuficiencia renal postoperatoria en pacientes sometidos a cirugía de revascularización miocárdica.

Métodos: estudio llevado a cabo entre enero de 2005 y diciembre de 2013, de casos y controles anidado en una cohorte, en la que se incluyeron pacientes sometidos a cirugía de revascularización miocárdica electiva que presentaron insuficiencia renal postoperatoria durante el postoperatorio inmediato hasta el egreso. Los controles estuvieron conformados por pacientes sometidos a cirugía de revascularización miocárdica electiva que no desarrollaron insuficiencia renal postoperatoria inmediata hasta el egreso. Se realizó un modelo de regresión logística para determinar los factores asociados a insuficiencia renal postoperatoria. Las asociaciones se expresaron en razones de disparidad con sus respectivos intervalos de confianza.

Resultados: la edad avanzada [OR 1,03 IC95\% $(1,01-1,04)$ ], la presencia preoperatoria de diabetes mellitus [OR 1,8 IC95\% (1,9-3,4)], la insuficiencia cardiaca [OR 2,7 IC 95\% $(1,1-6,7)$ ] y el mayor tiempo de perfusión [OR 1,02 IC 95\% (1,01-1,03)] se asociaron con mayor riesgo de insuficiencia renal postoperatoria, en tanto que el mayor hematocrito [OR 0,86 IC95\% $(0,82-0,91)$ ] y la mayor fracción de eyección [OR 0,94 IC95\% $(0,92-0,96)]$ se relacionaron con disminución del riesgo de insuficiencia renal postoperatoria.
\end{abstract}

\footnotetext{
* Autor para correspondencia.

Correos electrónicos: albert_barza@hotmail.com, albertbarza@gmail.com (A.F. Guerrero B.).
} 
Conclusiones: En quienes se realizó revascularización miocárdica los factores asociados a la presentación de insuficiencia renal postoperatoria fueron comorbilidades que se relacionaron con daño renal progresivo dentro y fuera del contexto de la cirugía. Esto implica que las estrategias para minimizar este evento estarán enfocadas a identificar de manera oportuna a estos pacientes y proporcionarles nefroprotección adecuada.

(c) 2015 Sociedad Colombiana de Cardiología y Cirugía Cardiovascular. Publicado por Elsevier España, S.L.U. Este es un artículo Open Access bajo la CC BY-NC-ND licencia (http:// creativecommons.org/licencias/by-nc-nd/4.0/).

\section{KEYWORDS}

Kidney failure;

Coronary

revascularisation

surgery;

Coronary disease

\section{Factors associated to postoperative kidney failure following myocardial revascularisation}

\begin{abstract}
Motivation: To determine which medical history and surgical procedure factors are associated to the onset of postoperative kidney failure in patients undergoing myocardial revascularisation. Methods: Case-control cohort study carried out between January 2005 and December 2013 which included patients who had undergone elective myocardial revascularisation and showed postoperative kidney failure immediately after surgery and until discharge. Controls consisted of patients who had undergone elective myocardial revascularisation and did not develop postoperative kidney failure immediately after surgery. A logistic regression model was used to determine the factors associated to postoperative kidney failure. Associations were expressed as grounds of disparity with their corresponding confidence intervals.

Results: old age [OR $1.03 \mathrm{Cl} 95 \%(1.01-1.04)]$, preoperative presence of diabetes mellitus [OR $1.8 \mathrm{Cl} 95 \%(1.9-3.4)]$, cardiac insufficiency [OR $2.7 \mathrm{CI} 95 \%(1.1-6.7)$ ] and a longer perfusion time [OR $1.02 \mathrm{Cl} 95 \%(1.01-1.03)]$ were associated to a higher risk of postoperative kidney failure, while higher hematocrit [OR $0.86 \mathrm{Cl} 95 \%(0.82-0.91)$ ] and higher ejection fraction [OR $0.94 \mathrm{Cl}$ $95 \%(0.92-0.96)]$ were associated with a decrease of the risk of postoperative kidney failure. Conclusions: In patients who had undergone myocardial revascularisation, risk factors associated to postoperative kidney failure where comorbidities related to internal and external gradual kidney damage outside the context of the surgery. This implies that strategies to minimise this event should be focused on identifying these patients in a timely manner and offering appropriate nephroprotection.

(c) 2015 Sociedad Colombiana de Cardiología y Cirugía Cardiovascular. Published by Elsevier España, S.L.U. This is an open access article under the CC BY-NC-ND license (http:// creativecommons.org/licenses/by-nc-nd/4.0/).
\end{abstract}

\section{Introducción}

La insuficiencia renal postoperatoria es una complicación de alta prevalencia y de importancia en la cirugía cardiaca. Se estima que más del $30 \%$ de los pacientes sometidos a cirugía cardiaca desarrolla insuficiencia renal postoperatoria clínicamente importante ${ }^{1,2}$. La Sociedad de Cirugía Torácica define la insuficiencia renal aguda postcirugía cardiaca como el aumento de creatinina sérica a $2 \mathrm{mg} / \mathrm{dl}$ o dos veces el valor preoperatorio, o nuevo requerimiento de hemodiálisis ${ }^{3,4}$.

En la Fundación Cardioinfantil-Instituto de Cardiología, la proporción de falla renal en el postoperatorio de cirugía cardiovascular es del 3,1\%. La aparición de insuficiencia renal postoperatoria en los pacientes sometidos a revascularización miocárdica se asocia con un aumento de la mortalidad, y en aquellos que requieren terapia dialítica el riesgo es ocho veces mayor ${ }^{1,2}$.

Para disminuir la insuficiencia renal después de revascularización miocárdica, se han efectuado múltiples intervenciones. Rannucci y Barret en diferentes estudios evaluaron el efecto del contraste del cateterismo y determinaron que no hay estudios suficientes que avalen el uso de n-acetil cisteína y bicarbonato, pero sí recomiendan la hidratación adecuada con solución salina y el uso de bajas dosis de contraste ${ }^{3,5}$. En Colombia, en el Hospital San Ignacio, se hizo un estudio para evaluar la aparición de lesión renal aguda después de cirugía cardiaca, que sugirió que los principales factores de riesgo no son modificables y debe hacerse énfasis en la nefroprotección preoperatoria ${ }^{6}$. Una estrategia razonable a realizar es la identificación de factores de riesgo modificables. Éstos podrían ser útiles como blancos terapéuticos para la prevención de disfunción renal. Por tal motivo, considerando que la Fundación Cardioinfantil-Instituto de Cardiología, es un centro especializado en cirugía cardiaca y no hay suficientes estudios en Colombia que aborden factores asociados a disfunción renal, se justifica hacer un estudio de factores de riesgo.

Este estudio se desarrolló con el propósito de determinar la incidencia de insuficiencia renal postoperatoria, describir los factores preoperatorios, intraoperatorios y postoperatorios asociados a su aparición y de esta manera poder identificarlos y planear estrategias y estudios para intervenir. Como hipóstesis se planteó que la presencia preoperatoria de anemia y diabetes mellitus, son factores que influyen en la aparición de insuficiencia renal posterior a cirugía de revascularización miocárdica. 


\section{Metodología}

Se realizó un estudio observacional, analítico, de tipo casos y controles anidado en una cohorte de pacientes sometidos a revascularización miocárdica. Ya que la insuficiencia renal postoperatoria en revascularización miocárdica es una complicación poco frecuente, se eligió este diseño que permite obtener información sobre causalidad de los factores asociados a dicha insuficiencia y a su vez determinar factores potencialmente modificables que ayuden a mejorar la seguridad del paciente.

El estudio se desarrolló en la Fundación CardioinfantilInstituto de Cardiología, con pacientes operados por el grupo de cirugía cardiovascular del adulto. Se realizó un muestreo no probabilístico de la cohorte de pacientes operados en el servicio de cirugía cardiovascular entre enero de 2005 y diciembre de 2013. En este periodo de tiempo se operaron 6.069 pacientes, de los cuales se seleccionaron aquellos que cumplieron los criterios de inclusión revascularización miocárdica como procedimiento aislado de primera vez y edad mayor a 18 años. Se excluyeron aquellos con disfunción renal preoperatoria (creatinina $>1,8 \mathrm{md} / \mathrm{dl}$ ), que fallecieran en sala de cirugía, en hemodiálisis, diálisis peritoneal o hemofiltración en el preoperatorio, sometidos a cirugía de urgencia y sin datos de creatinina pre- o postoperatoria. Para la selección de los casos de insuficiencia renal postoperatoria se aplicó la siguiente definición: pacientes que presentaron aumento mayor o igual a dos veces el valor de creatinina preoperatoria, durante el postoperatorio inmediato hasta el egreso. Para la selección del grupo control se incluyeron pacientes que no presentaron aumento de hasta dos veces el valor de creatinina preoperatoria durante el postoperatorio inmediato hasta el egreso. Tanto los casos como los controles fueron seleccionados desde el caso más reciente operado en diciembre de 2013, hasta el más antiguo en febrero de 2005. Los controles se eligieron de forma que quedaran proporcionalmente 4: 1 caso por año.

Para el cálculo de tamaño de la muestra se realizaron cálculos teniendo en cuenta las hipótesis del estudio. De todos los factores se escogió la prevalencia de anemia preoperatoria como el factor que más tamaño de muestra necesitaría para encontrar asociación con la presencia de insuficiencia renal postoperatoria.

La elección de los supuestos para el cálculo del tamaño de la muestra se obtuvo según los resultados de Karkouti et al., teniendo en cuenta los diferentes escenarios para los factores del estudio y que el tamaño de la muestra considerara el número de factores a evaluar ${ }^{1,6}$.

El cálculo se realizó en STATA 10.1 así: proporción exposición casos: 0,09; proporción exposición en controles: 0,04 ; relación control/caso: $4: 1$; error tipo । de $5 \%$ y poder del 80\%. Total casos: 197 ; total controles: 788 .

La definición de todas las variables siguió los lineamientos de la Society of Thoracic Surgeons STS Database Group para el registro de procedimientos cardiovasculares ${ }^{7}$. Las variables del estudio se clasificaron en tres grupos: características del paciente y del procedimiento quirúrgico.

El sesgo de selección de los pacientes del grupo control se controló obteniendo la población control y los casos de la misma cohorte de pacientes de cirugía de revascularización miocárdica, con una definición estándar que no ha cambiado en el tiempo y ha demostrado ser útil en pacientes de cirugía cardiovascular donde se obtuvieron los casos. Además se controló mediante la obtención de los casos y los controles en el mismo periodo de tiempo.

Los datos se obtuvieron directamente de la historia clínica en físico, en formato electrónico y en el registro institucional de laboratorios. Se consignaron en una base de datos independiente, registrando las variables clínicas del paciente, el procedimiento quirúrgico y el postoperatorio al igual que los desenlaces. Durante la limpieza de la base de datos se realizó la verificación de valores extremos en todas las variables cuantitativas, y se procedió de la misma forma con las variables de control creadas para las variables categóricas. Los valores extremos considerados por encima del percentil 95 o por debajo del percentil 5 se revisaron confrontando en la historia clínica los valores erróneos.

Se describió cada una de las variables del paciente, el procedimiento quirúrgico y el postoperatorio en general y para los grupos de casos y controles. Las variables continuas se describieron en medias o medianas con su respectiva medida de dispersión según la distribución de las variables. Las variables categóricas se describieron en frecuencias relativas y absolutas.

Se hizo un análisis bivariado para seleccionar las variables que se incluirían en el modelo inicial de regresión logística; para este paso se aceptaron valores de $\mathrm{p}<0,2$ para la selección de una variable. La asociación entre las variables y la insuficiencia renal postperatoria se estimó utilizando un modelo de regresión logística multivariada. Mediante un proceso de selección tipo stepwise, se evaluaron la interacción, la confusión y los diagnósticos de regresión para determinar que el modelo final cumpliera el criterio de jerarquía en relación con las variables incluidas.

Para establecer la exactitud del modelo se evaluaron la calibración y las pruebas de bondad de ajuste de Akaike y Bayesiana. Para la calibración se practicó la prueba de ajuste de bondad de Hosmer y Lemeshow $(\mathrm{H}-\mathrm{L})$.

Los análisis se procesaron en Stata 10,0 ; se consideraron estadísticamente significativos valores de $p<0,05$. La medida de asociación fue la razón de disparidad (OR) y su intervalo de confianza (IC) del 95\%.

Este estudio se condujo de acuerdo con los principios establecidos por la 18 a Asamblea Médica Mundial (Helsinki, 1964) y todas las enmiendas subsiguientes. Según la Resolución 008430 de 1993, artículo 11, del Ministerio de Salud de Colombia, se consideró investigación sin riesgo, motivo por el cual tampoco se firmó consentimiento; sin embargo, en el consentimiento informado del procedimiento que se usa en la Fundación Cardioinfantil-Instituto de Cardiología, existe un párrafo que describe el uso de la información del paciente para investigación en salud. Adicionalmente el estudio fue aprobado por los comités de Investigaciones y Ética de la Fundación Cardioinfantil-Instituto de Cardiología, y fue financiado con recursos propios del investigador.

\section{Resultados}

Entre enero de 2005 y diciembre de 2013 se realizaron 2.937 procedimientos de revascularización miocárdica aislada de primera vez. Para el análisis se incluyeron 192 casos 
Tabla 1 Características preoperatorias de los pacientes en los grupos de casos y controles

\begin{tabular}{|c|c|c|c|}
\hline Características & Controlesn $=788$ & Casosn= 192 & Valor $\mathrm{p}$ \\
\hline Edad media $\pm \mathrm{DE}$ & $64,3 \pm 9,8$ & $66,8 \pm 8,9$ & 0,001 \\
\hline Masculino n (\%) & $604(76,5)$ & $128(66,7)$ & 0,01 \\
\hline Peso $(\mathrm{kg})$ media $\pm \mathrm{DE}$ & $72,1 \pm 12,9$ & $70,3 \pm 11,9$ & 0,121 \\
\hline Talla $(\mathrm{cm})$ media $\pm D E$ & $166,0 \pm 8,5$ & $163,2 \pm 6,3$ & 0,001 \\
\hline$I M C$ media $\pm D E$ & $26,1 \pm 4,2$ & $26,3 \pm 4,0$ & 0,583 \\
\hline Hipercolesterolemia n (\%) & $462(58,6)$ & $120(62,5)$ & 0,327 \\
\hline Hipertensión arterial n (\%) & $606(76,9)$ & $164(85,4)$ & 0,010 \\
\hline Tabaquismo n (\%) & $310(39,3)$ & $72(37,5)$ & 0,226 \\
\hline Angioplastia previa n (\%) & $87(11,0)$ & $0(0)$ & 0,001 \\
\hline Infarto de miocardio n (\%) & $441(55,9)$ & $112(58,3)$ & 0,553 \\
\hline Accidente cerebrovascular n (\%) & $32(4,0)$ & $24(12,5)$ & 0,001 \\
\hline Arritmia n (\%) & $46(5,8)$ & $8(4,2)$ & 0,363 \\
\hline EPOC n (\%) & $54(2,6)$ & $2(6,7)$ & 0,18 \\
\hline EAOC $n(\%)$ & $75(3,7)$ & $5(16,7)$ & 0,0001 \\
\hline Diabetes mellitus n (\%) & $297(14,5)$ & $13(43,3)$ & 0,0001 \\
\hline \multicolumn{4}{|l|}{ Cirugía cardiaca previa n (\%) } \\
\hline Insuficiencia cardiaca n (\%) & $32(4,1)$ & $28(14,6)$ & 0,0001 \\
\hline Creatinina media $\pm \mathrm{DE}$ & $1,1 \pm 0,63$ & $1,2(0,9-1,7)$ & 0,182 \\
\hline Hematocrito media $\pm D E$ & $43,1 \pm 5,6$ & $39,3 \pm 7,1$ & 0,0001 \\
\hline Número de vasos media $\pm \mathrm{DE}$ & $3,2 \pm 1,1$ & $3,4 \pm 1,2$ & 0,111 \\
\hline Fracción de eyección media $\pm \mathrm{DE}$ & $49,6 \pm 10,9$ & $41,1 \pm 13,9$ & 0,0001 \\
\hline
\end{tabular}

MC: índice de masa corporal; EPOC: enfermedad pulmonar obstructiva crónica; EAOC: enfermedad arteria oclusiva cronica; DE: desviación estándar.

y 788 controles. Las características preoperatorias de los casos y los controles se observan en la tabla 1, mientras que en la tabla 2 se listan las características operatorias por grupo.

En la tabla 3 se observa el análisis bivariado con relación a la presencia de falla renal postoperatoria y en la tabla 4 el análisis multivariado.

Las variables que se asociaron con mayor presentación del evento fueron: edad, diabetes, enfermedad cerebrovascular, enfermedad arterial oclusiva crónica, hipertensión arterial, insuficiencia cardiaca congestiva, uso de circulación extracorpórea y tiempo de la misma o tiempo de perfusión.

Las variables que se asociaron con menor presentación del evento fueron: sexo masculino, hematocrito alto (punto de corte $33 \%$ ), fracción de eyección alta (punto de corte $55 \%)$ y uso de arteria mamaria interna.

En cuanto a las variables asociadas a insuficiencia renal postoperatoria se observó que la edad aumenta en 1,03 veces la posibilidad de insuficiencia renal postoperatoria (IC 95\% 0,99-1,06), un porcentaje de hematocrito alto disminuye en 0,86 veces la posibilidad de insuficiencia renal postoperatoria (IC 95\% 0,82-0,91), la diabetes mellitus aumenta en 1,8 veces la posibilidad de insuficiencia renal postoperatoria (IC 95\% 1,9-3,4), la presencia de enfermedad pulmonar aumenta en 1,3 veces la posibilidad de insuficiencia renal postoperatoria (IC 95\% 1,1-1,6), una fracción de eyección alta disminuye en 0,94 veces la posibilidad de insuficiencia renal postoperatoria (IC 95\% 0,92-0,96), el mayor tiempo de perfusión aumenta en 1,02 veces la posibilidad de insuficiencia renal postoperatoria (IC 95\% 1,01-1,03), la dislipidemia aumenta en 3,5 veces la posibilidad de insuficiencia renal postoperatoria (IC 95\% 1,7-7,1) y la presencia de insuficiencia cardiaca aumenta en 2,7 veces la posibilidad de insuficiencia renal postoperatoria (IC 95\% 1,1-6,7).

Se realizó un subanálisis teniendo en cuenta la presencia o ausencia de la variable sexo en el modelo de regresión multivariado; al realizar el análisis mediante la estrategia stepwise, la variable fue eliminada por no tener una asociación con el desenlace y no aportar al modelo. Sin embargo, fue incluida nuevamente al modelo final, obteniendo un AIC de 78 , un BIC de -2.526 y un LR de 30 , lo que indica un

Tabla 2 Características operatorias de los pacientes en los grupos de casos y controles

\begin{tabular}{llll}
\hline Características operatorias & Controlesn= 788 & Casosn= 192 & Valor $\mathrm{p}$ \\
\hline Uso de CEC $\mathrm{n}(\%)$ & $397(50,4)$ & $144(75)$ & 0,0001 \\
Tiempo de CEC media \pm DE & $89,7 \pm 25,4$ & $105,9 \pm 27,9$ & 0,0001 \\
Tiempo de pinza media \pm DE & $67,1 \pm 21,8$ & $80,1 \pm 21,1$ & 0,0001 \\
Mamaria izquierda & 88,3 & 89,6 & 0,79 \\
Mamaria bilateral & 0,25 & 0,0 & 0,07 \\
\hline
\end{tabular}

CEC: circulación extracorpórea; DE: desviación estándar. 
Tabla 3 Análisis bivariado para la presencia de falla renal postoperatoria

\begin{tabular}{|c|c|c|c|c|}
\hline \multirow{2}{*}{$\begin{array}{l}\text { Variables } \\
\text { Edad (años) }\end{array}$} & \multirow{2}{*}{$\begin{array}{l}\text { OR } \\
1,03\end{array}$} & \multirow{2}{*}{$\begin{array}{l}\text { Valor de } p \\
0,001\end{array}$} & \multicolumn{2}{|c|}{ IC $95 \%$} \\
\hline & & & 1,01 & 1,04 \\
\hline Sexo masculino & 0,61 & 0,005 & 0,43 & 0,85 \\
\hline Peso $(\mathrm{kg})$ & 0,98 & 0,122 & 0,97 & 1,00 \\
\hline Hematocrito (\%) & 0,89 & 0,001 & 0,87 & 0,92 \\
\hline Diabetes (si) & 2,28 & 0,0001 & 1,65 & 3,13 \\
\hline Enfermedad pulmonar (si) & 1,10 & 0,117 & 0,97 & 1,24 \\
\hline Enfermedad cerebrovascular (si) & 3,37 & 0,001 & 1,93 & 5,87 \\
\hline Hipertensión arterial (si) & 1,75 & 0,011 & 1,14 & 2,71 \\
\hline $\operatorname{ICC}(\mathrm{si})$ & 4,0 & 0,001 & 2,36 & 6,9 \\
\hline EAOC (si) & 2,17 & 0,007 & 1,24 & 3,81 \\
\hline Fracción de eyección (\%) & 0,94 & 0,001 & 0,92 & 0,95 \\
\hline Uso de CEC (si) & 2,9 & 0,001 & 2,0 & 4,3 \\
\hline Tiempo de perfusión (minutos) & 1,02 & 0,001 & 1,01 & 1,03 \\
\hline Uso de arteria mamaria interna (si) & 0,51 & 0,01 & 0,30 & 0,86 \\
\hline
\end{tabular}

ICC: insuficiencia cardiaca congestiva; EAOC: enfermedad arterial oclusiva crónica; CEC: circulación extracorpórea: OR: odds ratio; IC: intervalo de confianza.

detrimento en el ajuste del modelo ante la presencia de esta variable, motivo por el cual fue retirada del modelo.

Se realizaron los siguientes análisis de sensibilidad: presencia de reoperación por sangrado en el postoperatorio inmediato, presencia de hematocrito menor de $30 \mathrm{y}$ necesidad de hemoderivados con los factores asociados a insuficiencia renal postoperatoria asumiendo que los pacientes con estas características tenían un mayor riesgo de presentar insuficiencia renal postoperatoria; no obstante, al excluirlos no se observó una variación estadísticamente significativa en la asociación de las variables.

\section{Discusión}

A pesar del conocimiento de la fisiopatología de la insuficiencia renal postoperatoria, las estrategias usadas para

Tabla 4 Modelo de regresión logística para falla renal postoperatoria

\begin{tabular}{llr}
\hline Variable & \multicolumn{2}{c}{ Modelo final } \\
\cline { 2 - 3 } & OR & \multicolumn{1}{c}{ IC 95\% } \\
\hline Edad (años) & 1,03 & $0,99-1,06$ \\
Hematocrito (\%) & 0,86 & $0,82-0,91$ \\
Diabetes (si) & 1,8 & $1,9-3,4$ \\
Enfermedad pulmonar (si) & 1,3 & $1,1-1,6$ \\
Infarto de miocardio previo (si) & 0,57 & $0,30-1,05$ \\
Fracción de eyección (\%) & 0,94 & $0,92-0,96$ \\
Tiempo de perfusión (minutos) & 1,02 & $1,01-1,03$ \\
Dislipidemia (si) & 3,5 & $1,7-7,1$ \\
Insuficiencia cardiaca (si) & 2,7 & $1,1-6,7$ \\
LR & & 46,6 \\
Prob chi & & 0,000 \\
AIC & & 0,72 \\
AlB & & $-2471,81$ \\
\hline OR & &
\end{tabular}

OR: odds ratio; IC: intervalo de confianza; LR: likelihood ratio; AIC: bondad de ajuste de Akaike; AIB: bondad de ajuste Bayesiana. disminuir su aparición, la incidencia de insuficiencia renal postoperatoria y su mortalidad asociada siguen siendo altas. Esto se debe a que la mayoría de factores que la generan son no modificables, y por tanto se debe hacer énfasis sobre los modificables. La insuficiencia renal postoperatoria magnifica el riesgo de mortalidad luego de cirugía6,8-10. Se han usado múltiples estrategias de estratificación de riesgo para el diagnóstico y la intervención temprana de insuficiencia renal postoperatoria pese a las cuales no ha sido posible disminuir su aparición. Dentro de este grupo de pacientes, los sometidos a diálisis después de cirugía cardiaca presentaron mayor incidencia de infección, estancia prolongada en la unidad de cuidado intensivo y probabilidad de requerimiento de diálisis a largo plazo $0^{2,7,11}$. Se han clasificado los factores de riesgo en modificables y no modificables.

En este estudio observacional, analítico de tipo casos y controles, se analizaron solo pacientes sometidos a revascularización miocárdica electiva, y se excluyeron pacientes de alto riesgo, como aquellos sometidos a cirugía de emergencia, disfunción renal previa, hemodinámicamente inestables y con necesidad de balón de contrapulsación con el fin de identificar los factores asociados a disfunción renal en pacientes de bajo riesgo.

Chertow et al. identificaron en su escala de estratificación, el sexo femenino como un factor de riesgo para presentar insuficiencia renal postoperatoria, a diferencia de lo encontrado en nuestros resultados, ya que infortunadamente en nuestro caso el sexo femenino se comportó como un factor independiente; sin embargo en el modelo de regresión multivariado no se mantuvo la asociación ${ }^{12-15}$. Rajendre et al. en su escala de riesgo, identificaron la diabetes mellitus como un factor importante de insuficiencia renal postoperatoria, sobre todo en pacientes con control metabólico inadecuado, hallazgos que coinciden con los de nuestro estudio. Por tanto, se debe hacer un control metabólico preoperatorio estricto mediante el ajuste del manejo médico necesario para lograr glucometrías por debajo de $180 \mathrm{mg} / \mathrm{dl}$, apoyándose en un tratamiento interdisciplinario con el grupo de endocrinología y soporte nutricional ${ }^{7,8,15,16}$. Vargas et al. en el estudio realizado en 
el Hospital San Ignacio también evidenciaron la enfermedad pulmonar obstructiva crónica como factor de riesgo para insuficiencia renal postoperatoria ${ }^{6,17-19}$. La enfermedad vascular periférica se asocia a insuficiencia renal postoperatoria considerando que uno de los mecanismos fisiopatológicos de esta es la enfermedad ateroesclerótica presente en las arterias renales de este tipo de pacientes, de ahí que se deba llevar un control estricto de la presión arterial para evitar el deterioro endotelial progresivo. Karkouti et al. no evidenciaron la enfermedad vascular periférica como factor de riesgo; en su estudio hallaron un valor de $p=0,3^{20-22}$. La edad avanzada en nuestros pacientes casos, 66,8 $\pm 8,9$ años, demostró aumentar el riesgo de insuficiencia renal postoperatoria debido a posible esclerosis glomerular o enfermedad glomerular secundaria a otras noxas previas ${ }^{23,24}$. El hematocrito alto o la ausencia de anemia en nuestro estudio se presentaron como un factor protector de insuficiencia renal postoperatoria. Karkouti et al. determinaron, por su parte, que los pacientes anémicos que requirieron trasfusión de tres unidades de glóbulos rojos aumentaron el riesgo de insuficiencia renal postoperatoria de 1,8\% a 6,6\% y en los no anémicos que recibieron trasfusión incrementó de 1,7 a 3,2\%, por lo cual una estrategia para evitar la necesidad de trasfusión es el uso de salvador de células durante la cirugía ${ }^{25-27}$. La mayor duración de tiempo de CEC en nuestro grupo de casos se asoció con mayor riesgo de insuficiencia renal postoperatoria. La insuficiencia renal postoperatoria con CEC obedece a múltiples factores, entre ellos: disminución en la presión de perfusión renal, activación de mediadores proinflamatorios y nefrotoxicidad directa ${ }^{25,28,29}$. La CEC prolongada puede aumentar la hemólisis y la liberación de hemoglobina libre, la cual actúa como una toxina endógena al liberar hierro ${ }^{30}$. Todos estos hallazgos se han corroborado en los estudios de Karkouti, Duminda y Terrence ${ }^{1,10,31}$. La fracción de eyección conservada se asoció como factor protector para el desarrollo de insuficiencia renal postoperatoria, hallazgos similares en los estudios realizados por Chertow et al. La presencia de insuficiencia cardiaca congestiva genera hipoperfusión renal crónica secundaria al bajo gasto cardiaco, característica que hace a estos pacientes más susceptibles al daño renal durante el bajo flujo de la circulación extracorpórea, así que se precisa la optimización exhaustiva del manejo médico antes de llevar a cirugía ${ }^{32-34}$.

En este estudio se tuvieron limitaciones a considerar, como que fue de tipo retrospectivo, no multicéntrico, solo se tuvieron en cuenta los pacientes de bajo riesgo; por ser retrospectivo observacional no pueden tomarse decisiones con base en los resultados ni establecer la relación que hubo entre los medicamentos nefrotóxicos y el contraste usado en el cateterismo ya que no se dispuso de esta información en la base de datos; pese a ello nuestras estrategias consisten siempre en hidratar antes y después de cateterismo para lograr la mejor nefroprotección posible $e^{35-39}$.

\section{Conclusiones}

El estudio evidenció que en quienes se hará revascularización miocárdica de manera electiva, los factores no modificables con mayor efecto son la dislipidemia, la diabetes mellitus y la insuficiencia cardiaca congestiva por lo cual el control metabólico adecuado y el manejo médico apropiado podrían generar algún efecto sobre la aparición de insuficiencia renal postoperatoria.

Los factores modificables tales como el hematocrito preoperatorio adecuado son un factor protector para el desarrollo de insuficiencia renal postoperatoria, y se debe interactuar de manera agresiva sobre estos. El tiempo de perfusión es un factor modificable susceptible de mejoría a través de la estandarización de la técnica y la optimización de la planeación de la cirugía, para disminuir así la aparición de insuficiencia renal postoperatoria.

Dados los avances en medicamentos para el control de las comorbilidades de la enfermedad coronaria, técnicas operatorias y cuidados de perfusión en los pacientes sometidos a revascularización miocárdica, es posible que se necesiten estudios prospectivos para determinar la presencia de otros factores en los que podrán realizarse intervenciones a fin de menguar esta complicación.

\section{Responsabilidades éticas}

Protección de personas y animales. Los autores declaran que para esta investigación no se han realizado experimentos en seres humanos ni en animales.

Confidencialidad de los datos. Los autores declaran que en este artículo no aparecen datos de pacientes

Derecho a la privacidad y consentimiento informado. Los autores declaran que en este artículo no aparecen datos de pacientes

\section{Conflicto de intereses}

Los autores declaran no tener conflictos de intereses.

\section{Bibliografía}

1. Karkouti K, Wijeysundera DN, Yau TM, Callum JL, Cheng DC, Crowther $M$, et al. Acute kidney injury after cardiac surgery: focus on modifiable risk factors. Circulation. 2009;119:495-502.

2. Thakar CV, Yared JP, Worley S, Cotman K, Paganini EP. Renal dysfunction and serious infections after open-heart surgery. Kidney Int. 2003;64(1):239-46.

3. Ho AM, Chan SK. Renal dysfunction and CABG. Curr Opin Pharmacol. 2012;12(2):181-8.

4. Barkhordari K, Karimi A, Shafiee A, Soltaninia H, Khatami MR, Abbasi K, et al. Effect of pentoxifylline on preventing acute kidney injury after cardiac surgery by measuring urinary neutrophil gelatinase - associated lipocalin. J Cardiothorac Surg. 2011;19(6):8

5. Palomba H, de Castro I, Neto AL, Lage S, Yu L. Acute kidney injury prediction following elective cardiac surgery: AKICS score. Kidney Int. 2007;72(5):624-31.

6. Vargas J, Rodríguez M, Ruiz A. Predicción de aparición de injuria renal aguda después de cirugía cardiovascular en la unidad de cuidados intensivos del Hospital Universitario San Ignacio. Acta Médica Colomb. 2010;35(4):166-74.

7. Shahian DM, Jacobs JP, Edwards FH, Brennan JM, Dokholyan RS, Prager RL, et al. The Society of Thoracic Surgeons National Database. Heart. 2013.

8. Le Manach Y, Coriat P, Collard CD, Riedel B. Statin therapy within the perioperative period. Anesthesiology. 2008;108(6):1141-6. 
9. Lee SW, Kim WJ, Kim YH, Park SW, Park DW, Yun SC, et al. Preventive strategies of renal insufficiency in patients with diabetes undergoing intervention or arteriography. Am J Cardiol. 2011;107(10):1447-52.

10. Thakar CV, Arrigain S, Worley S, Yared JP, Paganini EP. A clinical score to predict acute renal failure after cardiac surgery. J Am Soc Nephrol. 2005;16(1):162-8.

11. Matsumoto M, Tanaka T, Yamamoto T, Noiri E, Miyata T, Inagi R, et al. Hypoperfusion of peritubular capillaries induced chronic hypoxia prior to progression of tubulointerstitial injury in a progressive model of rat glomerulonephritides. J Am Soc Nephrol. 2004;15(6):1574-81.

12. Dasta JF, Kane-Gill SL, Durtschi AJ, Pathak DS, Kellum JA. Costs and outcomes of acute kidney injury (AKI) following cardiac surgery. Nephrol Dial Transplant. 2008;23(6):1970-4.

13. Carcoana OV, Mathew JP, Davis E, Byrne DW, Hayslett JP, Hines $\mathrm{RL}$, et al. Mannitol and dopamine in patients undergoing cardiopulmonary bypass: A randomized clinical trial. Anesth Analg. 2003;97(5):1222-9.

14. Despotis G, Eby C, Lublin DM. A review of transfusion risks and optimal management of perioperative bleeding with cardiac surgery. Transfusion. 2008;48 $1 \mathrm{Suppl,} 2 \mathrm{~S}$-30S.

15. Coleman MD, Shaefi S, Coleman Sladen RN. Preventing acute kidney injury after cardiac surgery. Curr Opin Anaesthesiol. 2011;24(1):70-6.

16. National Kidney Foundation K/DOQI clinical practice guidelines for chronic kidney disease: Evaluation, classification, and stratification. Am J Kidney Dis. 2002;39(2 Suppl 1):S1-266.

17. Ranucci M, Ballotta A, Kunkl A, De Benedetti D, Kandil H, Conti $D$, et al. Influence of the timing of cardiac catheterization and the amount of contrast media on acute renal failure after cardiac surgery. Am J Cardiol. 2008;101(8):1112-8.

18. Patel NN, Toth T, Jones C, Lin H, Ray P, George SJ, et al. Prevention of post-cardiopulmonary bypass acute kidney injury by endothelin A receptor blockade. Crit Care Med. 2011;39(4):793-802.

19. Hashemzadeh K, Hashemzadeh S, Dehdilani M. Risk factors and outcomes of acute renal failure after open cardiac surgery. Asian Cardiovasc Thorac Ann. 2012;20(3):275-80.

20. Nangaku M. Chronic hypoxia and tubulointerstitial injury: A final common pathway to end-stage renal failure. J Am Soc Nephrol. 2006;17(1):17-25.

21. Murphy GS, Hessel EA, Groom RC. Optimal perfusion during cardiopulmonary bypass: An evidence based approach. Anesth Analg. 2009;108(5):1394-417.

22. Haase M, Haase-Fielitz A, Bellomo R, Devarajan P, Story D, Matalanis G, et al. Sodium bicarbonate to prevent increases in serum creatinine after cardiac surgery: A pilot doubleblind, randomized controlled trial. Crit Care Med. 2009;37(1): $39-47$.

23. Mangano CM, Diamondstone LS, Ramsay JG, Aggarwal A, Herskowitz A, Mangano DT. Renal dysfunction after myocardial revascularization: Risk factors, adverse outcomes, and hospital resource utilization. Ann Intern Med. 1998;128(3): 194-203.
24. Brezis M, Rosen S, Silva P, Epstein FH. Transport activity modifies thick ascending limb damage in the isolated perfused kidney. Kidney Int. 1984;25(1):65-72.

25. Vandenbroucke JP, von Elm E, Altman DG, Gøtzsche PC, Mulrow $\mathrm{CD}$, Pocock SJ, et al. STROBE Initiative. Strengthening the reporting of observational studies in epidemiology (STROBE): explanation and elaboration. Epidemiology. 2007;18(6):805-35.

26. Chawla LS, Zhao Y, Lough FC, Schroeder E, Seneff MG, Brennan JM. Off-pump versus on-pump coronary artery bypass grafting outcomes stratified by preoperative renal function. J Am Soc Nephrol. 2012;23(8):1389-97.

27. Arora P, Kolli H, Nainani N, Nader N, Lohr J. Preventable risk factors for acute kidney injury in patients undergoing cardiac surgery. J Cardiothorac Vasc Anesth. 2012;26(4):687-97.

28. Lal S, Murtagh JG, Pollock AM, Fletcher E, Binnion PF. Acute haemodynamic effects of furosemide in patients with normal and raised left atrial pressures. Br Heart J. 1969;31(6):711-7.

29. Mahesh B, Yim B, Robson D, Pillai R, Ratnatunga C, Pigott D. Does furosemide prevent renal dysfunction in high-risk cardiac surgical patients? Results of a double-blinded prospective randomised trial. Eur J Cardiothorac Surg. 2008;33(3):370-6.

30. Fisher AR, Jones P, Barlow P, Kennington S, Saville S, Farrimond $J$, et al. The influence of mannitol on renal function during and after open-heart surgery. Perfusion. 1998;13(3):181-6.

31. Poullis M. Mannitol and cardiac surgery. Thorac Cardiovasc Surg. 1999;47(1):58-62.

32. Sirivella S, Gielchinsky I, Parsonnet V. Mannitol, furosemide, and dopamine infusion in postoperative renal failure complicating cardiac surgery. Ann Thorac Surg. 2000;69(2):501-6.

33. Smith MN, Best D, Sheppard SV, Smith DC. The effect of mannitol on renal function after cardiopulmonary bypass in patients with established renal dysfunction. Anaesthesia. 2008;63(7):701-4.

34. Nielsen DV, Hjortdal V, Larsson H, Johnsen SP, Jakobsen CJ. Perioperative aminoglycoside treatment is associated with a higher incidence of postoperative dialysis in adult cardiac surgery patients. J Thorac Cardiovasc Surg. 2011;142(3):656-61.

35. González E, Gutiérrez E, Galeano C, Chevia C, de Sequera P, Bernis $C$, et al. Early steroid treatment improves the recovery of renal function in patients with drug induced acute interstitial nephritis. Kidney Int. 2008;73(8).

36. 16.Yoo YC, Shim JK, Kim JC, Jo YY, Lee JH, Kwak YL. Effect of single recombinant human erythropoietin injection on transfusion requirements in preoperatively anemic patients undergoing valvular heart surgery. Anesthesiology. 2011;115(5):929-37.

37. Li XM, Cong HL, Li TT, He LJ, Zhou YJ. Impact of benazepril on contrast-induced acute kidney injury for patients with mild to moderate renal insufficiency undergoing percutaneous coronary intervention. Chin Med J (Engl). 2011;124(14):2101-6.

38. Garcia S, Ko B, Adabag S. Contrast-induced nephropathy and risk of acute kidney injury and mortality after cardiac operations. Ann Thorac Surg. 2012;94(3):772-6.

39. Rydén L, Ahnve S, Bell M, Hammar N, Ivert T, Holzmann MJ. Acute kidney injury following coronary artery bypass grafting: early mortality and postoperative complications. Scand Cardiovasc J. 2012;46(2):114-20. 